\title{
ARTICLE
}

\section{A cyclic nucleotide-gated channel mediates cytoplasmic calcium elevation and disease resistance in rice}

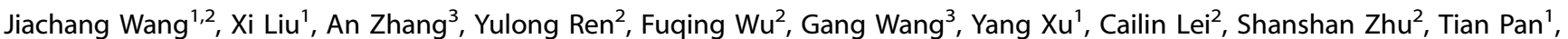 \\ Yongfei Wang ${ }^{1}$, Huan Zhang ${ }^{1}$, Fan Wang ${ }^{1}$, Yan-Qiu Tan ${ }^{3}$, Yupeng Wang ${ }^{2}$, Xin Jin ${ }^{2}$, Sheng Luo ${ }^{2}$, Chunlei Zhou ${ }^{1}$, Xiao Zhang ${ }^{1}$, Jinling Liu ${ }^{2}$, \\ Shuai Wang ${ }^{2}$, Lingzhi Meng ${ }^{2}$, Yihua Wang ${ }^{1}$, Xi Chen ${ }^{3}$, Qibing Lin ${ }^{2}$, Xin Zhang ${ }^{2}$, Xiuping Guo ${ }^{2}$, Zhijun Cheng ${ }^{2}$, Jiulin Wang ${ }^{2}$, Yunlu Tian ${ }^{1}$, \\ Shijia Liu ${ }^{1}$, Ling Jiang ${ }^{1}$, Chuanyin $\mathrm{Wu}^{2}$, Ertao Wang $\mathbb{D}^{3}$, Jian-Min Zhou (iD), Yong-Fei Wang $\mathbb{D}^{3}$, Haiyang Wang (D) ${ }^{2}$ and Jianmin Wan ${ }^{2}$
}

The transient elevation of cytoplasmic calcium is essential for pathogen-associated molecular pattern (PAMP)-triggered immunity (PTI). However, the calcium channels responsible for this process have remained unknown. Here, we show that rice CDS1 (CELL DEATH and SUSCEPTIBLE to BLAST 1) encoding OsCNGC9, a cyclic nucleotide-gated channel protein, positively regulates the resistance to rice blast disease. We show that OsCNGC9 mediates PAMP-induced $\mathrm{Ca}^{2+}$ influx and that this event is critical for PAMPs-triggered ROS burst and induction of PTI-related defense gene expression. We further show that a PTI-related receptor-like cytoplasmic kinase OsRLCK185 physically interacts with and phosphorylates OsCNGC9 to activate its channel activity. Our results suggest a signaling cascade linking pattern recognition to calcium channel activation, which is required for initiation of PTI and disease resistance in rice.

Cell Research (2019) 29:820-831; https://doi.org/10.1038/s41422-019-0219-7

\section{INTRODUCTION}

Plants are constantly challenged by various pathogenic pathogens (viruses, bacteria and fungi) and consequently, have evolved a two-tiered innate immune system to fend off pathogens: pathogen-associated molecular pattern (PAMP)-triggered immunity (PTI) and effector-triggered immunity (ETI). ${ }^{1,2} \mathrm{PTI}$ is generally considered to confer weaker, but broad-spectrum disease resistance, whereas $R$ gene-mediated ETI usually confers stronger race-specific disease resistance. ${ }^{3}$ PTI can be triggered via activation of plasma membrane (PM)-localized pattern recognition receptors (PRRs) by conserved pathogen-associated molecular patterns (PAMPs). Within minutes, pattern recognition triggers a set of signaling cascades, including increase of cytoplasmic calcium $\left(\left[\mathrm{Ca}^{2+}\right]_{\text {cyt }}\right)$, induction of reactive oxygen species (ROS) and expression of PTI-related defense genes. ${ }^{4}$ Further, it has been demonstrated that pattern-triggered $\left[\mathrm{Ca}^{2+}\right]_{\mathrm{cyt}}$ burst is implemented by $\mathrm{Ca}^{2+}$ influx from apoplast and $\mathrm{Ca}^{2+}$ release from intracellular stores. ${ }^{5}$ However, despite that $\mathrm{Ca}^{2+}$ influx has been demonstrated to be a core event for triggering $\mathrm{PTI}_{1}^{6,7}$ the channel proteins responsible for PAMP-mediated $\mathrm{Ca}^{2+}$ influx have remained elusive. ${ }^{8,9}$

On the other hand, it was recently shown that after pattern recognition, PRRs directly link with members of receptor-like cytoplasmic kinase subfamily VII (RLCK VII) to initiate multiple downstream signaling pathways. ${ }^{9}$ For example, Arabidopsis RLCK member BIK1 transmits signals from FLS2 by phosphorylation of
RbohD during PTI. $^{10}$ In rice, RLCK family members OsRLCK176 and OsRLCK185 interact with a rice PRR, OsCERK1, and are directly phosphorylated by OsCERK1 in a ligand-dependent manner. ${ }^{1-13}$ In line with this, the Osrlck176 mutant and Osrlck185 mutant show deficiencies in multiple pattern-induced immune responses, including ROS production, defense gene expression and MAPK activation. ${ }^{11,12}$ Although previous studies have shown that OsRLCK185 and OsRLCK176 mediate pattern-induced MAPK activation by interacting with OsCERK1, the underlying mechanisms by which rice RLCKs transmit signal to downstream signaling intermediates during PTI remain largely unclear.

Fungal pathogen Magnaporthe oryzae (M. oryzae) causes the most devastating disease that damages rice yield and grain quality worldwide. ${ }^{14}$ Several blast resistance genes encoding $\mathrm{R}$ proteins of the nucleotide-binding leucine-rich repeat (NLR) family have been identified, and they participate in ETI. ${ }^{15,16}$ On the other hand, accumulating evidence indicates PTI-mediated blast resistance also operates in rice. ${ }^{17-19}$ However, the signaling mechanisms governing PTI-mediated blast resistance in rice remain poorly understood.

The cyclic nucleotide-gated channels (CNGCs) gene family encodes a group of nonspecific, $\mathrm{Ca}^{2+}$-permeable cation channels. ${ }^{20}$ Several Arabidopsis CNGCs (AtCNGCs) have been characterized electrophysiologically. For example, AtCNGC1 and AtCNGC2 are permeable to both $\mathrm{K}^{+}$and $\mathrm{Ca}^{2+}, 21,22$ and AtCNGC5, $6,7,8,9,10,14,16$ and 18 have been shown to be $\mathrm{Ca}^{2+}$-permeable diverlant cation-selective channels. ${ }^{23-29}$ In rice,

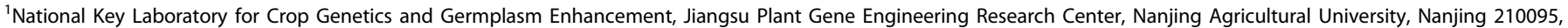

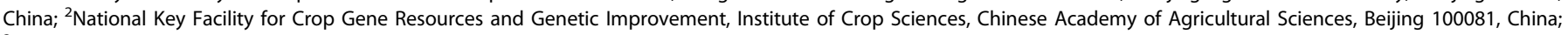

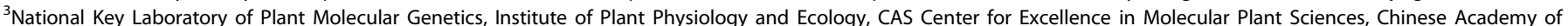

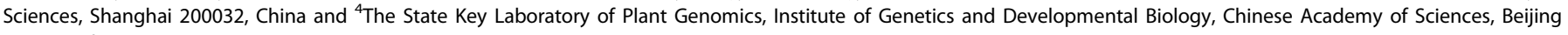
100101, China

Correspondence: Jianmin Wan (wanjm@njau.edu.cn)

These authors contributed equally: Jiachang Wang, Xi Liu, An Zhang

Received: 25 June 2019 Accepted: 1 August 2019

Published online: 23 August 2019 
OsCNGC13 was also identified as a $\mathrm{Ca}^{2+}$-permeable diverlant cation-selective channel. ${ }^{30}$ CNGCs have been shown to be involved in numerous physiological processes in plants, including immune responses, pollen tube germination and elongation, stomatal movement and root gravitropism. ${ }^{31}$ For example, the Arabidopsis defense-no-death (dnd1) mutant was identified as a mutant for AtCNGC2 and the constitutive expresser of pathogenesisrelated (PR) genes 22 (cpr22) mutant was shown to be caused by a genomic deletion, resulting in an in-frame chimeric AtCNGC11AtCNGC12 fusion. ${ }^{32,33}$ Although the effects of CNGCs on R-genemediated and damage associated molecular pattern (DAMP)induced immune responses have been reported, little is known about their role in plant PTI signaling pathway. ${ }^{8}$

In this study, we report the isolation and mechanistic characterization of a rice mutant, cds1 (cell death and susceptible to blast 1), which displayed impaired blast resistance at the seedling stage. CDS1 encodes a rice CNGC protein, OsCNGC9. The OsCNGC9mediated $\mathrm{Ca}^{2+}$ influx is important for PAMPs-induced signaling events, including ROS burst and PTI-related gene expression. Further analysis indicates that rice PTI signal transducer, OsRLCK185, physically interacts with and phosphorylates OsCNGC9 to activate its channel activity. Together, these findings provide novel mechanistic insights into the regulatory mechanisms of OsCNGC9 and suggest a signaling cascade linking PAMPs recognition to OsCNGC9-mediated $\mathrm{Ca}^{2+}$ influx to trigger PTI in plants.

\section{RESULTS}

The cds1 mutant plants show impaired blast resistance at the seedling stage and lesion-mimic phenotype after flowering In order to identify new genes involved in the regulation of rice disease resistance, we isolated a stable natural mutant $c d s 1$ with enhanced susceptibility to rice blast from the progenies derived from the cross of two japonica cultivars, Ningjing1 and Yun2273. Although cds1 seedlings were completely normal in morphology (Supplementary information, Fig. S1a), blast resistance assay revealed that they were significantly more susceptible to rice blast than wild type (WT) seedlings. After inoculating with blast, the lesion length and relative fungal growth of punch-inoculated leaves of WT seedlings were obviously lower than those of $c d s 1$ seedlings (Fig. 1a-c). Interestingly, cds1 leaves developed spontaneous cell death, examined by trypan blue staining (an indicator of programmed cell death, $P C D$ ), and measurement of chlorophyll contents after flowering (Supplementary information, Fig. S1b-f). qRT-PCR analysis showed that the expression of a representative cell death marker gene (OsNAC4) was significantly up-regulated in the cds 1 mutants (Supplementary information, Fig. S1g). These observations suggest that $C D S 1$ is a positive regulator of rice innate immunity at the seedling stage, but its genetic lesion can trigger spontaneous PCD after flowering, a phenomenon common to many important immune proteins. ${ }^{1,34}$

\section{CDS1 encodes a CNGC}

Genetic analysis revealed that the lesion mimic phenotype of $c d s 1$ was controlled by a single recessive nuclear gene (Supplementary information, Table S1). The CDS1 locus was mapped to a 40-kb genomic region flanked by the markers PCD-22 and PCD-27 using a large F2 mapping population developed by crossing $c d s 1$ with the indica cultivar 9311 (Fig. 1d). Sequence analysis revealed a 4-bp deletion in the coding region of LOC_Os09g38580, but no mutation was detected in other genes contained in the fine mapping region (Fig. 1e). LOC_Os09g38580 was predicted to encode a cyclic nucleotide-gated channel with six transmembrane domains, a pore-forming region and a CNBD domain at the C-terminal region (Fig. 1f) and it was previously named OsCNGC9. ${ }^{35}$ The 4-bp deletion was predicted to introduce a premature stop codon, resulting in translation of only the first 5 transmembrane domains in cds1 (designated OsCNGC9-cds1 hereafter) (Fig. 1f).
To verify that LOC_Os09g38580 corresponds to the CDS1 gene, we performed a genetic complementation assay. The transgene including the entire coding region of LOC_Os09g38580 with its native promoter ( $p G O s C N G C 9$ ) fully rescued the impaired blast resistance phenotype at the seedling stage and the spontaneous cell death phenotype of $c d s 1$ after flowering in all positive transgenic lines (Fig. 1g-i, Supplementary information, Fig. S1h-k). These results confirmed that LOC_Os09g38580 (OsCNGC9) is the underlying gene for CDS1.

The expression pattern and protein properties of OsCNGC9 qRT-PCR analysis showed that OsCNGC9 was expressed in all tissues examined, with relatively higher expression in leaves (Supplementary information, Fig. S2a). The expression pattern was further verified by histochemical staining of the transgenic rice carrying a $\beta$-glucuronidase (GUS) reporter driven by the endogenous OsCNGC9 promoter (Supplementary information, Fig. S2b-g). Further, the preferential expression of OsCNGC9 in leaf mesophyll cells was confirmed using RNA in situ hybridization (Supplementary information, Fig. S2h, i).

Multiple sequence alignment and phylogenic analysis showed that OsCNGC9 is conserved in both dicots and monocots (Supplementary information, Fig. S3a, b). Transient expression of the P35S:OsCNGC9-GFP construct in the rice protoplasts showed that OsCNGC9 was exclusively localized to the plasma membrane (Supplementary information, Fig. S4a-d). Similar result was also observed in stable transgenic rice expressing OsCNGC9-GFP driven by the OsCNGC9 native promoter (Supplementary information, Fig. S4e-h). Yeast two-hybrid $(\mathrm{Y} 2 \mathrm{H})$ assay and bimolecular fluorescence complementation (BiFC) assay revealed that OsCNGC9 could form homo-complex (Supplementary information, Fig. S4i, j), consistent with the earlier report that some CNGC isoforms can form homo-complex in planta. ${ }^{36}$

OsCNGC9 is a $\mathrm{Ca}^{2+}$-permeable divalent cation-selective inward channel

To analyze the ion permeability and selectivity of OsCNGC9, we first tested the accumulation of 9 different ions in Escherichia coli (E. coli) transiently expressing OsCNGC9. The contents of $\mathrm{Ca}^{2+}$, $\mathrm{Na}^{+}, \mathrm{K}^{+}, \mathrm{Mg}^{2+}, \mathrm{Mn}^{2+}, \mathrm{Fe}^{2+}, \mathrm{Cu}^{2+}, \mathrm{Zn}^{2+}$ and $\mathrm{Ni}^{2+}$ were measured using inductively coupled plasma atomic-emission spectroscopy (ICP-AES). The results showed that the relative intracellular contents of $\mathrm{Na}^{+}, \mathrm{K}^{+}$and $\mathrm{Mg}^{2+}$ were decreased lightly, whereas the content of $\mathrm{Ca}^{2+}$ was significantly increased in $E$. coli expressing OsCNGC9 relative to the control (Fig. 2a), suggesting that OsCNGC9 could be $\mathrm{Ca}^{2+}$ selective relative to other cations. In support of this notion, intracellular $\mathrm{Ca}^{2+}$ accumulation was completely abolished by the application of $100 \mu \mathrm{M}$ external gadolinium $\left(\mathrm{Gd}^{3+}\right)$, a well-known $\mathrm{Ca}^{2+}$ channel blocker (Fig. 2b). We next tested the external $\left[\mathrm{Ca}^{2+}\right]$ - and time-dependence of intracellular $\mathrm{Ca}^{2+}$ accumulation in $E$. coli, and observed strong intracellular $\mathrm{Ca}^{2+}$ accumulation in an external $\left[\mathrm{Ca}^{2+}\right]-$ and timedependent manner (Fig. 2c, d). Similar cytosolic $\mathrm{Ca}^{2+}$ increase was observed in intact HEK293T cells expressing wild type OsCNGC9, but not in HEK293T cells expressing OsCNGC9-cds1, upon the application of $10 \mathrm{mM}$ external $\mathrm{CaCl}_{2}$ (Fig. 2e, f). Additionally, whole-cell patch clamping experiments showed large inward channel currents in HEK293T cells expressing wild type OsCNGC9 in $10 \mathrm{mM} \mathrm{Ca}^{2+}$ bath solution, but only tiny background conductance was observed in HEK293T cells expressing OsCNGC9-cds1 and the mock control under the same condition (Fig. 2g, h). Notably, we observed that the large OsCNGC9-mediated inward whole-cell currents were abolished by removal of external $10 \mathrm{mM} \mathrm{Ca}^{2+}$ (Supplementary information, Fig. S5a, b), demonstrating a clear dependence of external $\mathrm{Ca}^{2+}$ for OsCNGC9-mediated inward whole-cell currents. However, the whole-cell currents were relatively small $(<100 \mathrm{pA})$, which precluded us from accurately measuring the shift of reversal 


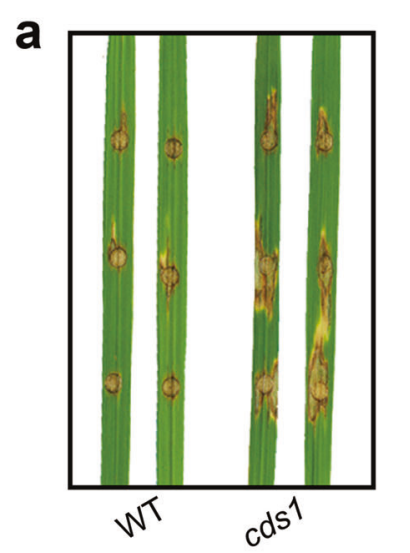

b

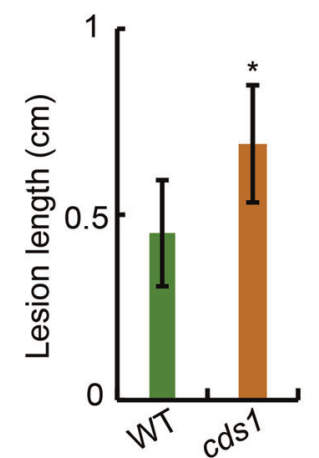

C

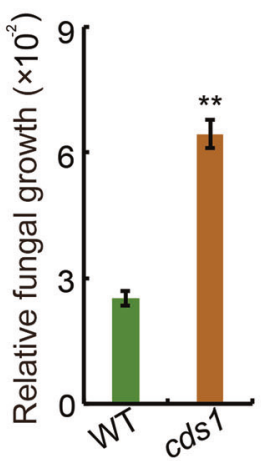

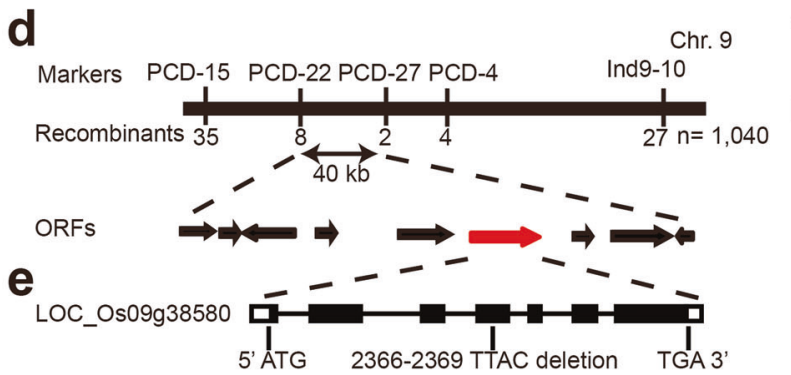
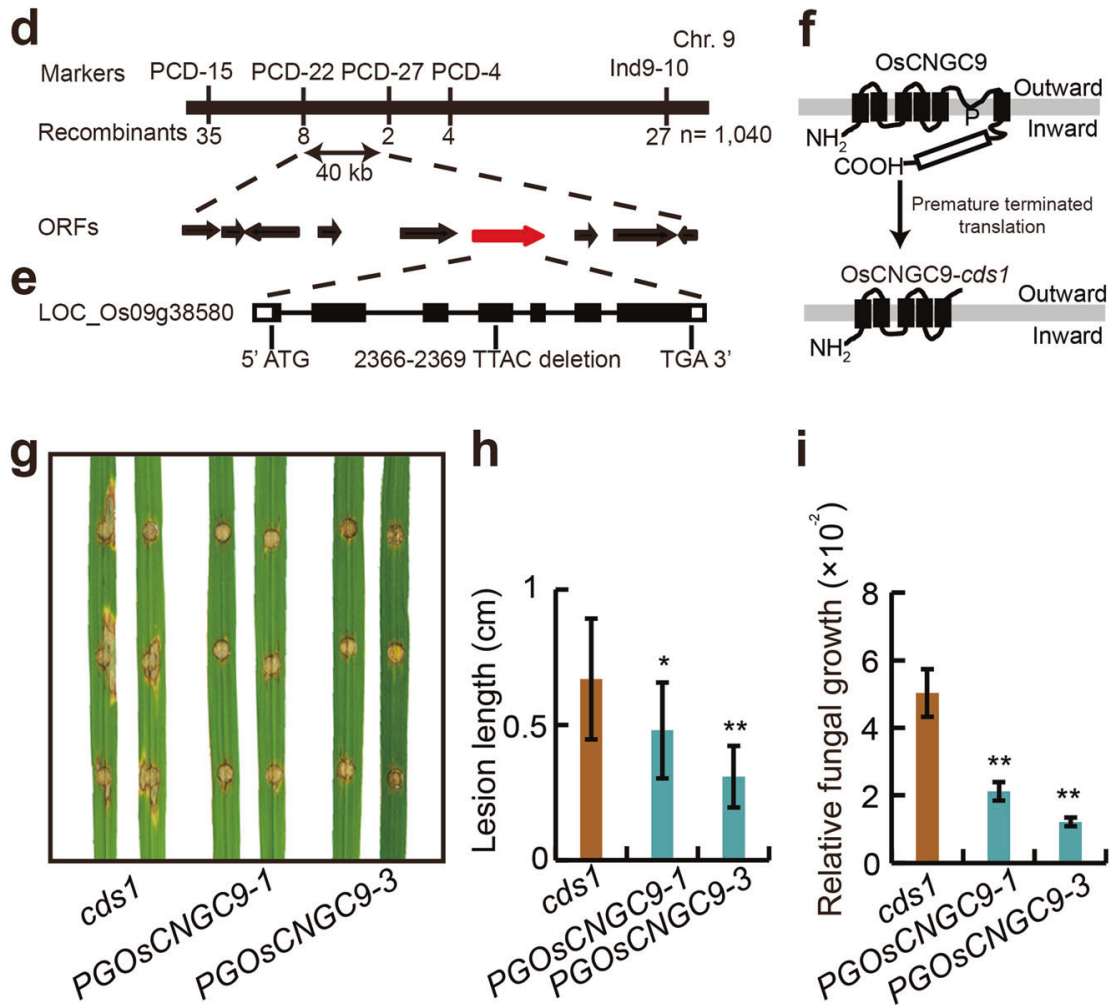

Fig. 1 The mutation in OsCNGC9 is responsible for impaired blast resistance. a The cds 1 mutant plants are more susceptible to blast at the seedling stage (blast isolate 2015-412 was used for inoculation). b, c Lesion length (b) and relative fungal growth (c) of WT and cds1 plants after punch inoculation with the blast. d Fine mapping of OsCNGC9. e Gene structure and the mutation site in OsCNGC9. f Schematic diagram of OsCNGC9 secondary structure prediction. $\mathbf{g}$ pGOsCNGC9 rescues the seedling blast susceptibility phenotype of the cds 1 plants. $\mathbf{h}$, $\mathbf{i}$ Lesion length (h) and relative fungal growth (i) of $c d s 1$ and two pGOsCNGC9 transgenic lines after inoculation with the blast. WT, a normal sibling segregated from the stable progeny of the same cross between japonica Ningjing1 and japonica Yun2273. Values are means \pm SD ( $\mathbf{b}, \mathbf{h}, \mathbf{n}=10$ samples; $\mathbf{c}, \mathbf{i}, \mathrm{n}=3$ replicates). WT control is only shown in (a) for simplicity. The Student's $t$-test analysis indicates a significant difference $\left({ }^{*} P<0.05,{ }^{* *} P<0.01\right)$

potential upon the change of external $\mathrm{Ca}^{2+}$ concentration. Thus, we further tested the effect of $\mathrm{Ca}^{2+}$ channel blockers $\left(\mathrm{La}^{3+}\right.$ and $\left.\mathrm{Gd}^{3+}\right)$, and found that the application of either one of these two $\mathrm{Ca}^{2+}$ channel blockers could completely abolish OsCNGC9mediated inward whole-cell currents in $10 \mathrm{mM} \mathrm{Ca}^{2+}$ bath solution (Supplementary information, Fig. S5c, d). Together, these results demonstrate that OsCNGC9 functions as an inward $\mathrm{Ca}^{2+}$ channel, and the mutation in cds 1 disrupts its calcium channel activity.

It has been reported that some plant plasma membrane $\mathrm{Ca}^{2+}$-permeable channels show similar permeability to $\mathrm{Ba}^{2+}$ and $\mathrm{Ca}^{2+24}$ we thus analyzed the permeability of OsCNGC9 to $\mathrm{Ba}^{2+}$. We replaced $10 \mathrm{mM} \mathrm{Ca}^{2+}$ in the bath solution with $\mathrm{Ba}^{2+}$ of the same concentration, and observed large inward channel currents in HEK293T cells expressing OSCNGC9 (Supplementary information, Fig. S5e, f), similar to OsCNGC9-mediated large inward currents recorded in $10 \mathrm{mM} \mathrm{Ca}^{2+}$ bath solution. Considering that some AtCNGCs are permeable to both $\mathrm{Ca}^{2+}$ and $\mathrm{K}^{+}, 21$ we also tested whether OsCNGC9 is permeable to monovalent cations. Patch clamping experiments in HEK293T cells using $\mathrm{K}^{+}$-based bath and pipette solutions showed large inward $\mathrm{K}^{+}$channel currents in HEK293T cells expressing the well-characterized typical inward $\mathrm{K}^{+}$ channel AtKAT1, ${ }^{37}$ but only a tiny background conductance in HEK293T cells expressing OsCNGC9 (Supplementary information, Fig. S5g, h). Thus, OsCNGC9 does not have obvious permeability to monovalent $\mathrm{K}^{+}$. 

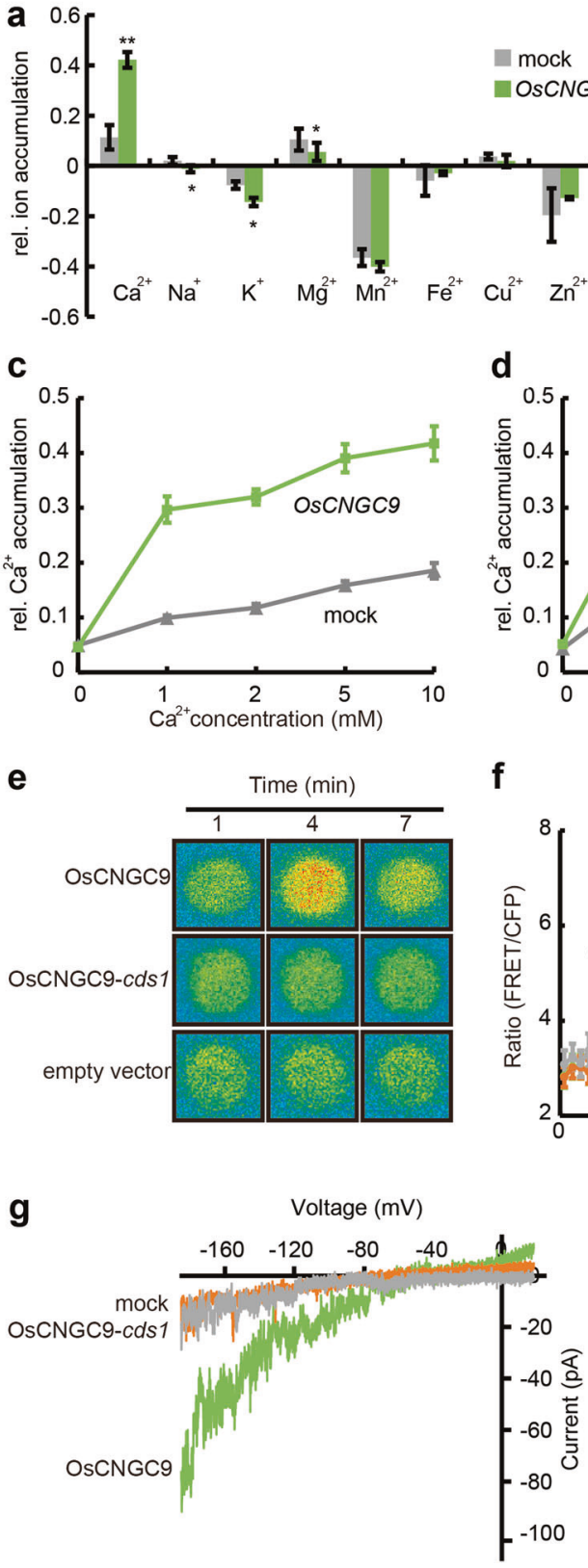
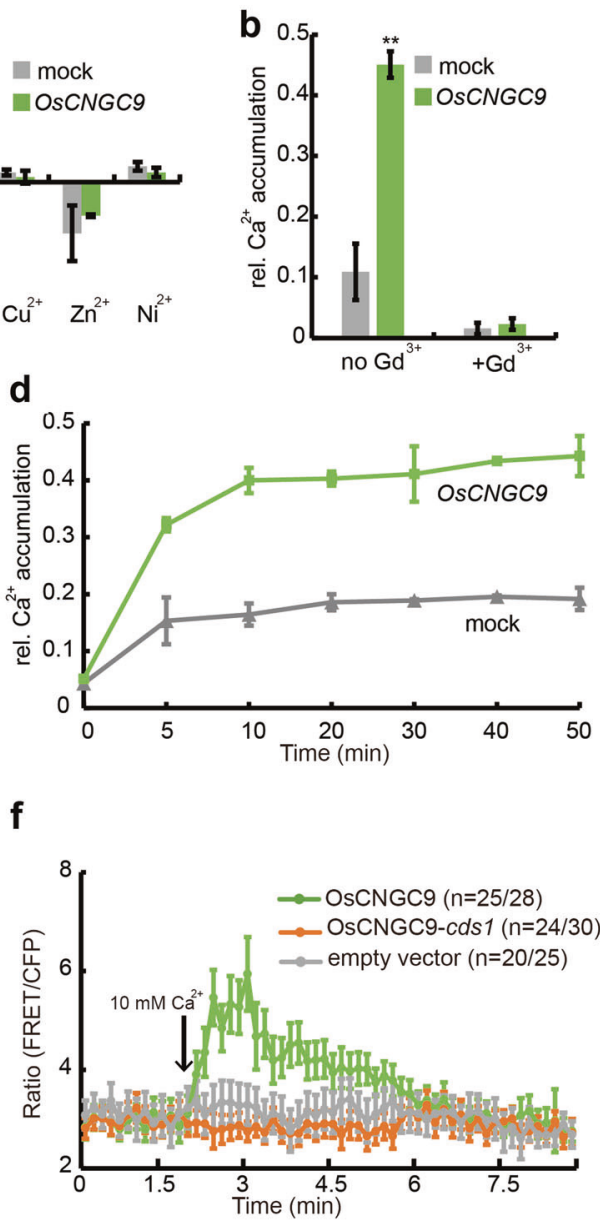

f

h Voltage $(\mathrm{mV})$

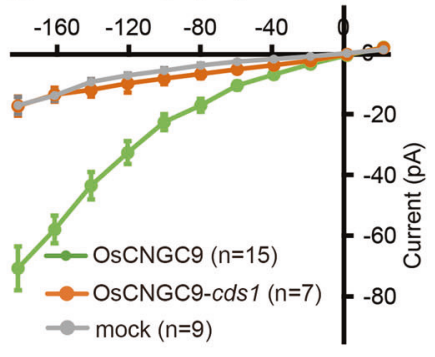

Fig. 2 OsCNGC9 is a $\mathrm{Ca}^{2+}$-permeable divalent cation-selective inward channel. a ICP-AES measurement of the intracellular contents of various ions in E. coli expressing OsCNGC9. b Accumulation of intracellular $\mathrm{Ca}^{2+}$ in $E$. coli expressing OsCNGC9 after calcium channel blocker Gd ${ }^{3+}(0.1$ $\mathrm{mM}$ ) treatment. c, d The external $\left[\mathrm{Ca}^{2+}\right]$-dependent (c) and time-dependent (d) accumulation of intracellular $\mathrm{Ca}^{2+}$ in $E$. coli expressing OsCNGC9. e, f Typical fluorescent images (e) and average fluorescent intensity changes (f) in HEK293T cells expressing OsCNGC9 or OsCNGC9cds1. The two numbers in parentheses indicate the number of cells with the representative calcium pattern versus the number of total cells analyzed. $\mathbf{g}, \mathbf{h}$ Typical whole-cell recordings (g) and the average current-voltage relationship (h) of patch clamping results in HEK293T cells expressing OsCNGC9 or OsCNGC9-cds1. Values are means \pm SD (a-d, $\mathrm{n}=3$ replicates). The Student's $t$-test analysis indicates a significant difference $(* P<0.05, * * P<0.01)$

OsCNGC9 is required for $\mathrm{Ca}^{2+}$ influx, ROS burst and PTI-related genes expression in response to PAMPs

We next investigated whether OsCNGC9 can mediate $\mathrm{Ca}^{2+}$ influx in PTI by measuring the dynamics of $\mathrm{Ca}^{2+}$ flux in mesophyll cells after treatment with two PAMPs (chitin or flg22) in vivo using a Non-invasive Micro-test Technique. Previous studies have shown that these PAMP elicitors can trigger PTI signaling in plants. ${ }^{38,39}$ In response to chitin or flg22 stimulation, WT mesophyll cells, but not cds1 mesophyll cells, exhibited robust and fast $\mathrm{Ca}^{2+}$ influx (Fig. 3a, $\mathrm{b}$ and Supplementary information, Fig. S6). These results suggest that OsCNGC9 can mediate $\mathrm{Ca}^{2+}$ influx in rice PTI and this ability is impaired in the cds 1 mutants.
Pattern-induced $\mathrm{Ca}^{2+}$ influx precedes and is required for a number of signaling events including ROS burst in $\mathrm{PTI}^{40}$ We thus used a chemical luminescence assay to test whether PAMPstriggered ROS burst is impaired in the cds 1 mutants. The ROS production level was higher in WT leaves than that in cds1 after treatment with chitin or flg22. ROS level peaked about $11 \mathrm{~min}$ after chitin or flg22 treatments (Fig. 3c, d). In addition, qRT-PCR analysis showed that in response to flg22 or chitin treatments, the induction levels of two PTI-related defense genes (KS4 and PAL) were notably lower in the cds1 mutants compared to WT plants (Fig. 3e, f). These results suggest that OsCNGC9 participates in the regulation of rice $\mathrm{PTI}$. 

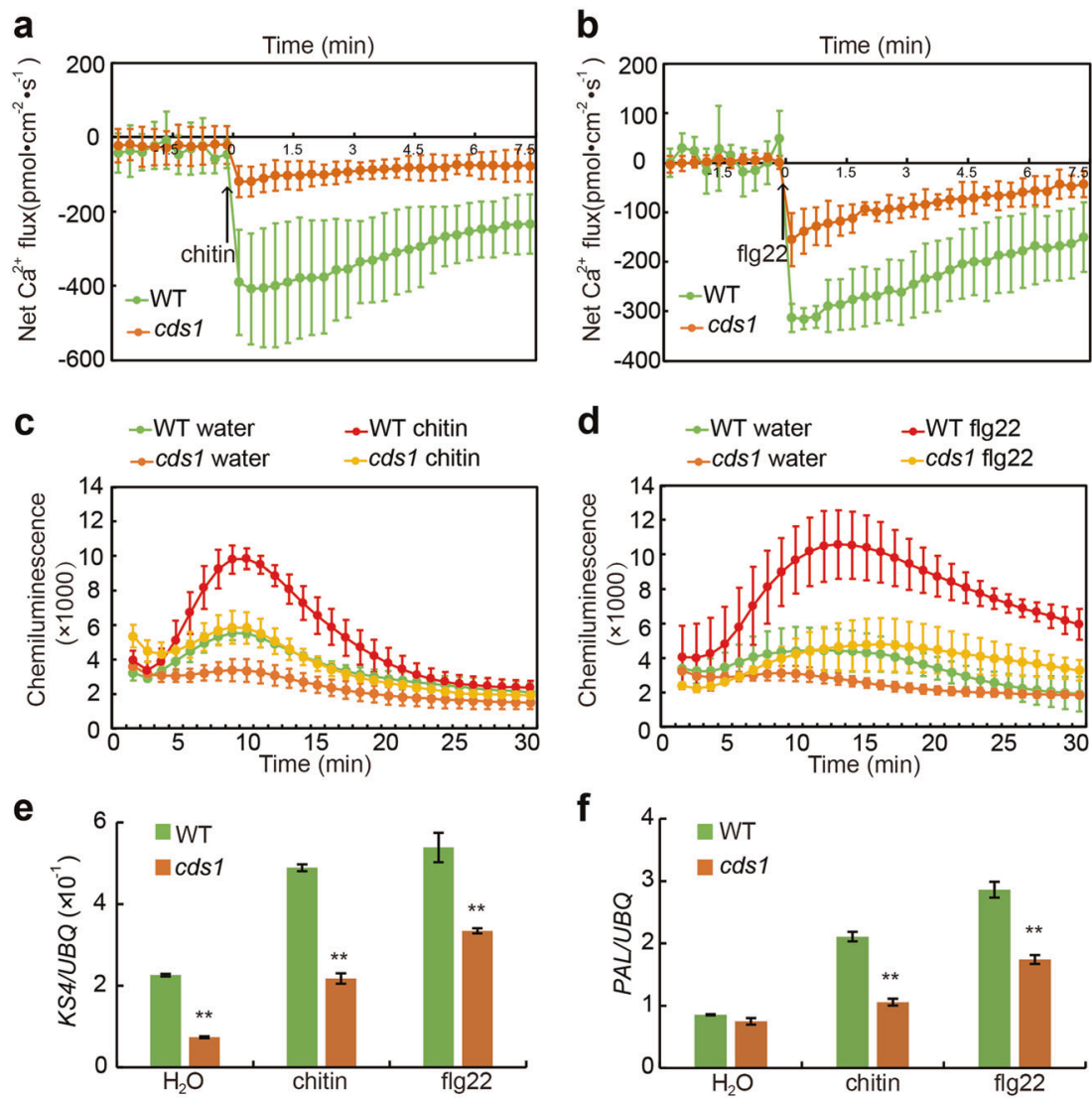

Fig. 3 OsCNGC9 is required for $\mathrm{Ca}^{2+}$ influx, ROS burst and PTI-related defense genes expression in response to PAMPs. a, b Comparison of calcium influx in mesophyll cells from WT and cds1 plants after chitin (a) or flg22 (b) treatments. c, d Dynamic analysis of chitin (c) and flg22induced (d) ROS generation in WT and cds1 leaves. e, $\mathbf{f}$ The expression levels of two PTI-related defense genes (KS4 and PAL) after treatment with chitin or flg22 in WT and cds1 plants. Values are means \pm SD (a, $\mathbf{b}, \mathrm{n}=6$ replicates; $\mathbf{c}-\mathbf{f}, \mathrm{n}=3$ replicates). The Student's $t$-test analysis indicates a significant difference $\left({ }^{* *} P<0.01\right)$

OsRLCK185 physically interacts with and phosphorylates OsCNGC9 to activate its channel activity

Recent studies have shown that PRRs and members of RLCK VII play important roles in regulating plant innate immunity. ${ }^{9}$ To investigate the functional relationship between OsCNGC9 and PRRs/RLCKs, we performed yeast two-hybrid (Y2H) assay and luciferase complementation assay to test potential protein-protein interaction between OsCNGC9 with four rice PRRs and two RLCKs that have been previously reported to be involved in PTI. ${ }^{4}$ Both assays showed that OsCNGC9 could interact with three of them, including OsFLS2, OsRLCK176 and OsRLCK185 (Fig. 4a, b and Supplementary information, Fig. S7a, b). It has been shown that RLCKs, BIK1 and PBL1 act upstream of calcium channels in Arabidopsis. ${ }^{10,41} \mathrm{Y} 2 \mathrm{H}$ assay showed that OsCNGC9 interacts with OsRLCK176 and OsRLCK185 via its C-terminus (Supplementary information, Fig. S7c, d). Thus, we examined whether OsRLCK176 and OsRLCK185 can phosphorylate OsCNGC9 using an in vitro phosphorylation assay. The results showed that only OsRLCK185 could effectively phosphorylate the C-terminus of OsCNGC9 (Fig. 4C). These results suggest that OsRLCK185 is likely a regulator of OsCNGC9 during rice PTI. In support of this notion, coimmunoprecipitation assay confirmed the interaction between OsCNGC9 and OsRLCK185 in rice protoplasts (Fig. 4d).

We next investigated the effect of OsRLCK185 on the $\mathrm{Ca}^{2+}$ channel activity of OsCNGC9 expressed in HEK293T cells. A stronger cytosolic $\mathrm{Ca}^{2+}$ increase was observed in intact HEK293T cells coexpressing OsCNGC9 and OsRLCK185 upon application of $10 \mathrm{mM}$ external $\mathrm{CaCl}_{2}$, but not in intact HEK293T cells coexpressing OsCNGC9 and OsRLCK185-K108E (an inactive form of OsRLCK185 ${ }^{12}$ ) (Fig. 4e). In addition, no significant cytosolic $\mathrm{Ca}^{2+}$ increase was observed in the HEK293T cells expressing either OsRLCK185 or OsRLCK185-K108E alone (Supplementary information, Fig. S7e). To further verify pattern-induced phosphorylation of OsCNGC9 C-terminus in vivo, we developed a phos-tag assay. A chitin-induced band-shift of the C-terminal fragment of OsCNGC9 was observed in wild type (Kitaake) protoplasts, but not in Oscerk1 knockout mutant protoplasts (Fig. $4 \mathrm{f}$ and Supplementary information, Fig. S7f). These data collectively suggest that phosphorylation of OsCNGC9 by OsRLCK185 leads to activation of the OsCNGC9 channel activity and $\mathrm{Ca}^{2+}$ influx in response to PAMP.

Osrlck185/55 double mutant plants show impaired blast resistance and reduced pattern-induced $\mathrm{Ca}^{2+}$ influx

We next tested whether OsRLCK185 plays a role in regulating rice blast resistance. To avoid potential functional redundancy, ${ }_{12}^{12}$ we conducted a blast resistance assay using Osrlck185 and Osrlck55 (a close homologous gene of OsRLCK185, which shares $84 \%$ amino acid identity with OsRLCK185 ${ }^{42}$ ) double mutant plant (Fig. 5a). After inoculating with blast, the lesion length and relative fungal growth of punch-inoculated leaves of Osrlck185/55 double mutant seedlings were obviously higher than those of the corresponding wild type (Nipponbare) seedlings (Fig. 5b-d). $\mathrm{Ca}^{2+}$ flux assay showed that in response to chitin stimulation, Nipponbare mesophyll cells, but not Osrlck185/55 double mutant mesophyll cells, exhibited fast $\mathrm{Ca}^{2+}$ influx (Fig. 5e). Moreover, no significant $\mathrm{Ca}^{2+}$ influx was observed after chitin treatment of the Oscerk1 knockout mutant (Fig. 5f). These results together suggest that 
a

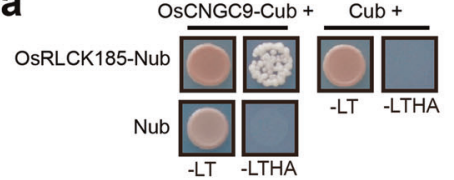

b

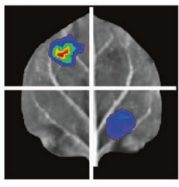

CLUC-OSCNGC9 CLUC-OSCNGC9 nLuc-OSRLCK185 nLuc-GW5 \begin{tabular}{l|l}
\hline cLuc-GW5 & cLuc-OsCNGC9
\end{tabular} nLUC-OSRLCK185 nLUC-OSCNGC9 High $L$ Low

e

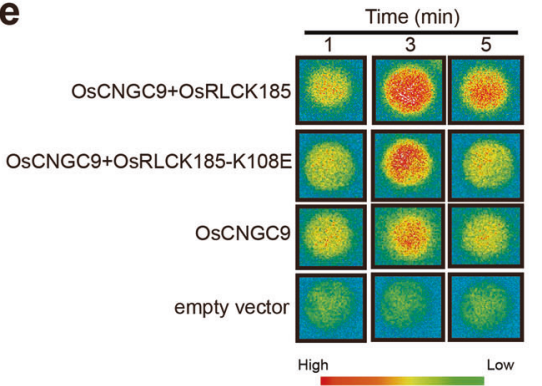

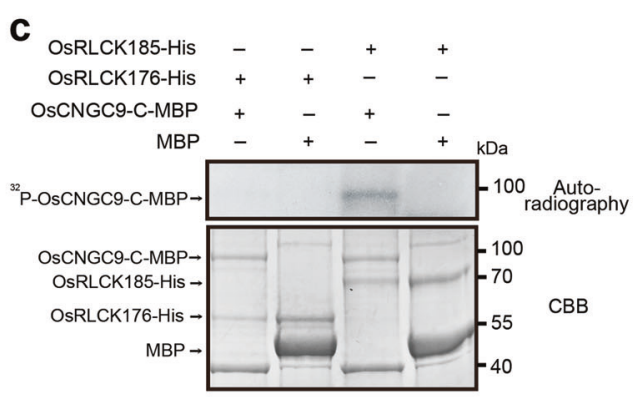

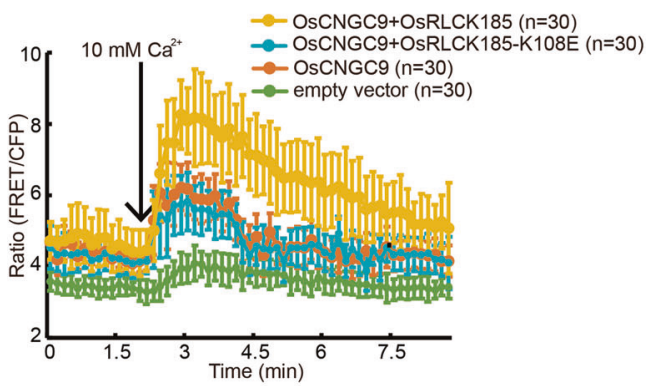

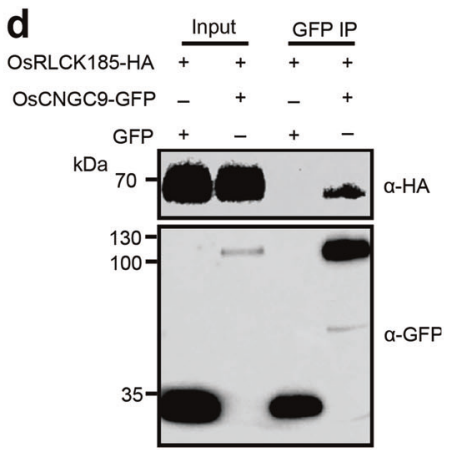

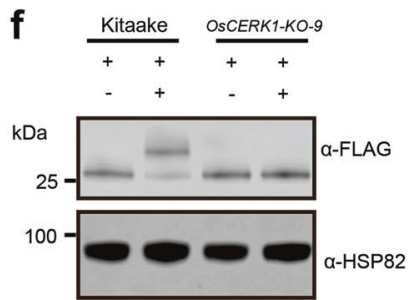

Fig. 4 OsRLCK185 physically interacts with and phosphorylates OsCNGC9 to activate its channel activity. a OsCNGC9 interacts with OsRLCK185 in Y2H assay. b OsCNGC9 interacts with OsRLCK185 in firefly luciferase complementation assay. c In vitro phosphorylation assay showing that the C-terminus of OsCNGC9 (OsCNGC9-C) is phosphorylated by OsRLCK185. d Co-immunoprecipitation assay shows that OsCNGC9 interacts with OsRLCK185 in rice protoplasts. e Ca ${ }^{2+}$ imaging experiments showing the activation of OsCNGC9 by OsRLCK185 and the consequent increase of $\left[\mathrm{Ca}^{2+}\right]_{\text {cyt }}$ in HEK293T cells. Typical fluorescent images (left panel) and average fluorescent intensity changes (right panel) in HEK293T cells transiently expressing various tested proteins as indicated. $\mathbf{f}$ Chitin induces phosphorylation of OsCNGC9 C-terminus. Kitaake or OsCERK-KO-9 protoplasts expressing OsCNGC9-C-FLAG were treated with chitin for 10 min before protein extraction. Total protein was fractionated by SDS-PAGE containing Phos-tag acrylamide (upper panel) and normal SDS-PAGE (lower panel)

a OsRLCK185
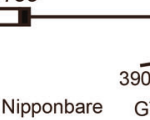
303

Nipponbare

GAAGCACTTGATCGC - AATGGACTTCAAGGAAA OSRLCK55

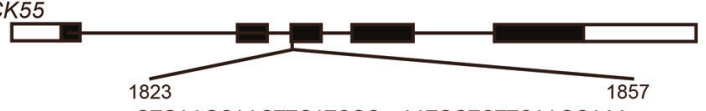

Nipponbare GTGAAGCAACTTGATCGC - AATGGTCTTCAAGGAAA OsRLCK185/55-KO-1 GTGAAGCAACTTGATCGC CAATGGTCTTCAAGGAAA +1bp

b

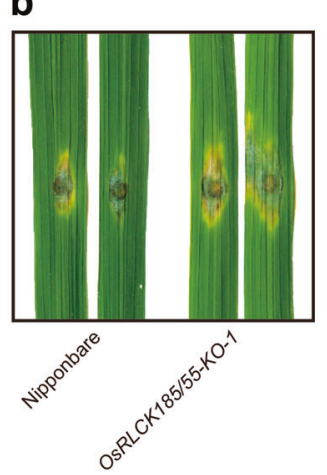

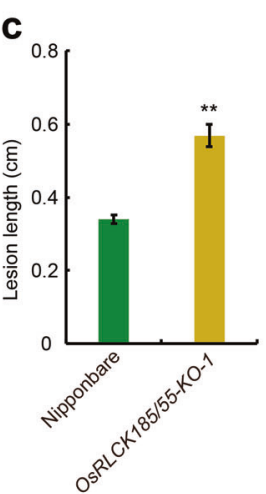
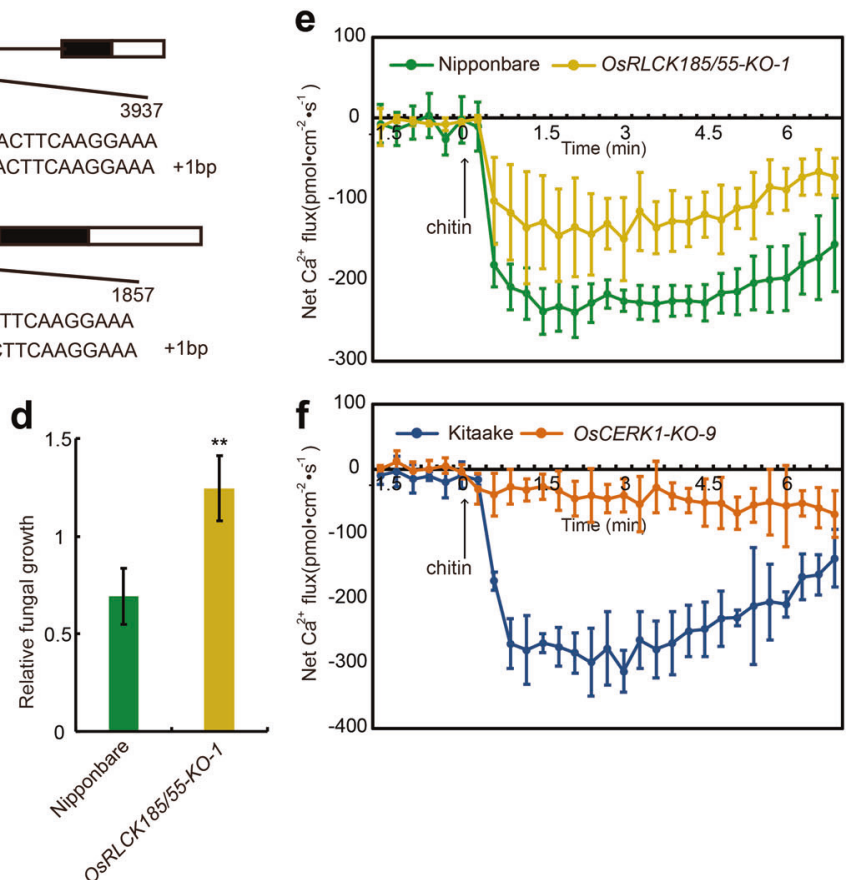

Fig. 5 Osrlck185/55 double mutant plants show impaired blast resistance and reduced chitin-induced Ca ${ }^{2+}$ influx. a Sketch map of the mutations in the knockout line (Osrlck185/55-KO-1). Plus (+) sign indicates the number of nucleotides. b The Osrlck185/55 double mutant plants are more susceptible to blast at the seedling stage (blast isolate 2015-412 was used for inoculation). c, d Lesion length (c) and relative fungal growth (d) of Nipponbare and Osrlck185/55 double mutant plants after punch inoculation with the blast. e Comparison of calcium influx in mesophyll cells from Nipponbare and Osrlck185/55 double mutant plants after chitin treatment. f Comparison of calcium influx in mesophyll cells from Kitaake and Oscerk1 mutant plants after chitin treatment. Values are means \pm SD $(\mathbf{c}, \mathrm{n}=10$ samples; $\mathbf{d}, \mathrm{n}=3$ replicates; $\mathbf{e}, \mathbf{f}, \mathrm{n}=6$ replicates). The Student's $t$-test analysis indicates a significant difference $\left({ }^{* *} P<0.01\right)$ 
a

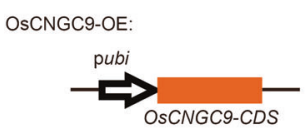

b

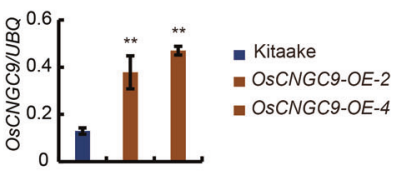

C
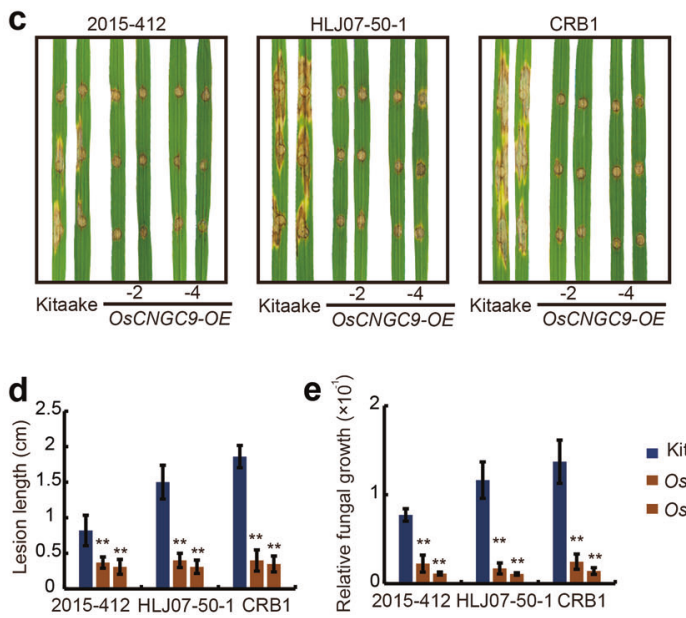

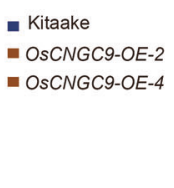

f

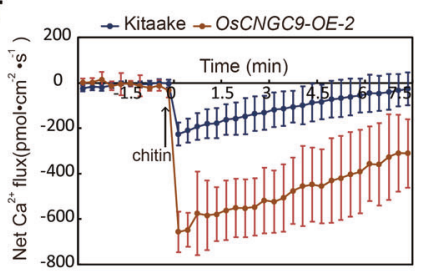

h $\rightarrow$ Kitaake water $\rightarrow$ OSCNGC9-OE-2 water $\rightarrow$ Kitaake chitin $\rightarrow$ OSCNGC9-OE-2 chitin

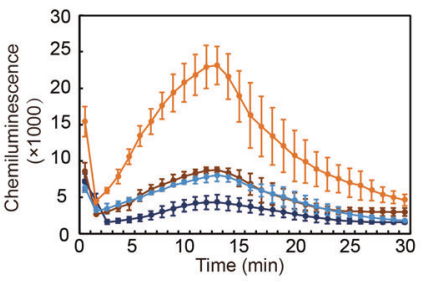

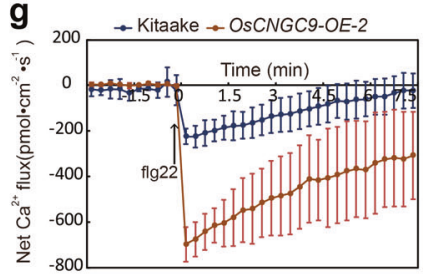

\section{i}

$\rightarrow$ Kitaake flg22 $\rightarrow$ OSCNGC9-OE-2 flg22

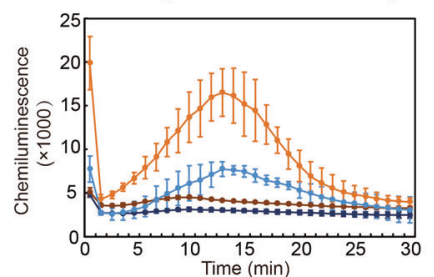

Fig. 6 OsCNGC9 expression positively regulates rice PTI and blast resistance. a Diagram of the OsCNGC9-OE construct. Pubi, maize Ubiquitin promoter; OsCNGC9-CDS, the coding sequence of OsCNGC9. b qRT-PCR measurement of the expression levels of OsCNGC9 in leaves of Kitaake and the OsCNGC9-OE transgenic plants. c The OsCNGC9-OE transgenic lines confer increased blast resistance. Three blast isolates (2015-412, HLJ07-50-1 and CRB1) were used in the assay. d, e Lesion length (d) and relative fungal growths (e) were recorded in Kitaake and OsCNGC9-OE transgenic lines after inoculation with blast isolates. f, $\mathbf{g}$ Comparison of calcium influx in the mesophyll cells of Kitaake and OsCNGC9-OE transgenic plants after chitin (f) or flg22 (g) treatments. $\mathbf{h}$, i Comparison of ROS induction in the leaves of Kitaake and OsCNGC9-OE transgenic plants subject to treatment of chitin (h) or flg22 (i) for $1 \mathbf{h}$. j, k Comparison of the expression levels of $K S 4$ (j) and PAL (k) after flg22 or chitin treatments in leaves of Kiaake and OsCNGC9-OE transgenic plants. Values are means \pm SD (d, $\mathrm{n}=10$ samples; $\mathbf{b}, \mathbf{e}-\mathbf{k}, \mathrm{n}=3$ replicates). The Student's $t$-test analysis indicates a significant difference $(* * P<0.01)$

OsRLCK185 and its close homologue, OsRLCK55, participate in the regulation of blast resistance and PAMP-induced $\mathrm{Ca}^{2+}$ influx in rice.

OsCNGC9 overexpression confers enhanced rice PTI and blast resistance

To evaluate the potential value of OsCNGC9 in crop genetic improvement, we generated OsCNGC9 overexpression (OsCNGC9$O E)$ transgenic lines by transforming the coding sequence of OsCNGC9 under control of the maize UBIQUITIN promoter into Kitaake background (Fig. 6a). Two stable transgenic lines with increased levels of the OsCNGC9 transcripts were identified and selected for further blast resistance assay (Fig. 6b). We found that the OsCNGC9-OE lines conferred increased blast resistance to three different blast isolates, compared with the Kitaake (Fig. 6c-e). Consistently, after PAMPs stimulation, the mesophyll cells of OsCNGC9-OE transgenic plants showed stronger $\mathrm{Ca}^{2+}$ influx, higher PAMPs-triggered ROS burst and higher levels of PTIrelated defense genes expression compared with those of Kitaake plants (Fig. 6f-k). Therefore, we conclude that OsCNGC9 functions as a rate-limiting positive regulator of rice PTI.

\section{DISCUSSION}

In summary, here we identify OsCNGC9 as a positive regulator of PTI, which acts as a $\mathrm{Ca}^{2+}$-permeable divalent cation-selective inward channel. We further show that OsCNGC9 channel activity can be activated by OsRLCK185-mediated phosphorylation. Together with the earlier report that OsRLCK185 is phosphorylated by the chitin receptor OsCERK1 during chitin-induced plant immunity, ${ }^{12,13}$ our data collectively suggest a model in which OsCERK1and OSRLCK185 are activated upon pattern recognition, leading to phosphorylation and enhancement of OsCNGC9 channel activity. Then OsCNGC9-mediated $\mathrm{Ca}^{2+}$ influx triggers a series of immune responses including ROS burst and PTI-related defense genes expression, which ultimately leads to enhanced disease resistance in rice (Fig. 7). In support of this model, we showed that chitin-induced phosphorylation of OsCNGC9 Cterminus and chitin-induced $\mathrm{Ca}^{2+}$ influx were severely impaired in the Oscerk1 knockout mutant (Figs. 4f, $5 \mathrm{f}$ and Supplementary information, Fig. S7f). Moreover, phosphorylation of human CNGC has also been shown to be involved in regulating channel activity. ${ }^{43}$

It is worth mentioning that besides OsRLCK185, several other OsRLCKs have been shown to be involved in regulation of PTI responses and disease resistance in rice. ${ }^{9,18}$ Their regulatory relationship with OsCERK1 and OsCNGC9 will be an interesting avenue for future research. In addition, there is also evidence suggesting that the activation of MAPK cascades and anion channel opening are also triggered by pattern recognition. ${ }^{9,44}$ Thus, the relationship between OsCNGC9-mediated $\mathrm{Ca}^{2+}$ influx and PAMPs-triggered signaling events in immune responses is also worthy investigation in the future. Moreover, we observed that both ROS level and PTI-related gene expression were lower in cds 1 compared to WT without PAMPs treatment (Fig. 3), suggesting that OsCNGC9 may also play additional roles unrelated to PTI. In line with this, AtCNGC14, a close homologue of OsCNGC9, was reported to regulate root development. ${ }^{27,45}$

Like mutant plants with mutations of many immune proteincoding genes, ${ }^{1,34}$ cds 1 mutant plants display an autoimmunity phenotype including spontaneous $P C D$, enhanced $P R$ gene expression and enhanced disease resistance after flowering (Supplementary information, Fig. S8). Similar phenotypes were also observed in the Arabidopsis dnd 1 mutant, which is defective 


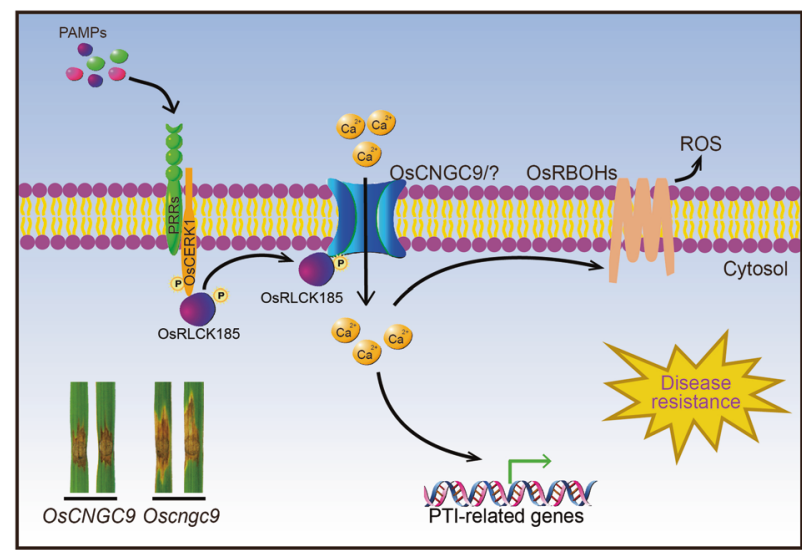

Fig. 7 A proposed model for OsCNGC9 in regulating rice PTI. PAMPs, such as the flg22 and chitin, are sensed by pathogen recognize receptors (PRRs), leading to the activation of OsRLCK185 by OsCERK1. ${ }^{12,13}$ Subsequently, the activated OsRLCK185 phosphorylates OsCNGC9 to enhance its channel activity. The activated OsCNGC9 induces extracellular $\mathrm{Ca}^{2+}$ influx and then triggers ROS burst and PTI-related gene expressions, which ultimately leads to enhanced disease resistance in rice. "OsCNGC9/?" represents the potential homomeric and/or heteromeric tetramers that contains OsCNGC9

in AtCNGC2.32 It is believed that these proteins have become targets of pathogen effectors during host-pathogen co-evolution. Plant NLR proteins are known to guard these effector targets (guardees) and to initiate immunity when these proteins are posttranslationally modified by effectors or altered by genetic lesions in their coding genes. ${ }^{46}$ Thus, the autoimmunity phenotypes of cds 1 plants may result from developmental stage-dependent activation of an unknown NLR that guards OsCNGC9, a possibility to be tested in the future.

Diseases cause significant crop loss, which is a serious threat to global food security. Crop genetic improvement is considered to be a better strategy against diseases than pesticide application that causes environmental pollution. Our result that overexpression of OsCNGC9 significantly enhanced rice blast resistance (Fig. 6) suggests that precise regulation of OsCNGC9 expression level may be a useful strategy to balance yield potential and enhanced disease resistance in rice.

\section{MATERIALS AND METHODS}

Plant materials and growth conditions

The cds 1 mutant was initially identified from the progenies of a cross between japonica Ningjing1 and japonica Yun2273, which displayed a cell death phenotype after flowering in the paddy field. Wild type is a normal sibling segregated from the stable progeny of the same cross between japonica Ningjing1 and japonica Yun2273. The cds1 mutant, japonica Kitaake and Nipponbare were used for further transgenic analysis. All plants including transgenic rice plants were grown in the isolated paddy fields during natural growing seasons at the Chinese Academy of Agricultural Sciences (Beijing) or in a controlled growth chamber $\left(14 \mathrm{~h}\right.$ light at $30^{\circ} \mathrm{C} / 10 \mathrm{~h}$ darkness at $25^{\circ} \mathrm{C}$, relative humidity of $\sim 80 \%$ and light intensity of $\sim 800 \mu \mathrm{mol} \mathrm{m}^{-2} \mathrm{~s}^{-1}$ ) for phenotypic analysis and pathogen inoculation.

Blast inoculation on rice leaves

A punch inoculation method with slight modifications was used to inoculate rice plants with blast isolates as described previously. ${ }^{47}$ Briefly, blast isolates were cultured on oat meal agar medium for two weeks for generating spores. Spores were collected by washing the fungal agar cultures with sterile water (containing $1 \%$ Tween 20, Sigma), and the spore concentration of blast isolates was adjusted to $\sim 5 \times 10^{5}$ spore/ml with sterile water (containing $1 \%$ Tween 20) before punch inoculation. Detached Leaves of seedling or 7 DAF rice plants were wounded with a hole-punch. $8 \mu \mathrm{l}$ blast spore suspension was applied to the injured area, and then the inoculated leaves were placed in sterile water that contains $0.1 \%$ 6-Benzylaminopurine (Sigma) to keep the moist. The inoculated leaves were kept in darkness at $28^{\circ} \mathrm{C}$ for $24 \mathrm{~h}$ before they were transferred to a controlled growth chamber $(12 \mathrm{~h}$ light at $28^{\circ} \mathrm{C} / 12 \mathrm{~h}$ darkness at $28^{\circ} \mathrm{C}$ ). Lesion length was measured using a ruler when significant lesion difference was observed. Relative fungal growth (MoPot/UBQ) was measured using qRT-PCR. All primer sequences used here are listed in Supplementary information, Table S2.

Leaf PCD assay

For trypan blue staining assay, leaves of rice plants on $7 \mathrm{DAF}$ were submerged in lactic acid-phenol-Trypan blue solution (25\% lactic acid, $23 \%$ water-saturated phenol, $0.25 \%$ Trypan blue and $25 \%$ glycerol) for $48 \mathrm{~h}$. Stained leaf samples were incubated in $70 \%$ ethanol until the tissue became clear. For cell death marker gene expression assay, OsNAC4 was selected as cell death marker gene for detecting leaf PCD level.

The TUNEL assay was performed as described by $\mathrm{Xu}$ et al. ${ }^{30}$ Briefly, leaves were fixed in $4 \%$ paraformaldehyde, dehydrated in an ethanol series and embedded in wax. Paraffin-embedded leaves were cut and hydrated and then treated with proteinase $K$ in PBS for $20 \mathrm{~min}$ at $37^{\circ} \mathrm{C}$ in a humid chamber, followed by rinsing in PBS for three times. The TUNEL assay was conducted with a Dead End Fluorometric TUNEL Kit (Promega) following the manufacturer's instructions. A laser scanning confocal microscope (ZEISS LSM 700) was used to detect the TUNEL signal. The green fluorescence of fluorescein (TUNEL signal) was analyzed at $488 \mathrm{~nm}$ (excitation) and $520 \mathrm{~nm}$ (detection). The red fluorescence of propidium iodide was analyzed at $488 \mathrm{~nm}$ (excitation) and $610 \mathrm{~nm}$ (detection).

\section{Map-based cloning}

To map the CDS1 gene, 1,182 mutant individuals were collected from the $F_{2}$ population of $c d s 1$ crossed with 9311 . The CDS1 locus was firstly mapped to a $300-\mathrm{kb}$ region on chromosome 9 using $142 \mathrm{~F}_{2} c d s 1$ individuals by molecular markers. Next, the $c d s 1$ locus was further mapped to a 40-kb region using 1,040 $F_{2} \quad c d s 1$ individuals and newly developed molecular markers. PCR and sequencing analysis were employed to identify the candidate gene. All primer sequences used for the map-based cloning procedure are listed in Supplementary information, Table S2.

Plasmid construction and plant transformation

For complementation construct, a 6.5 -kb genomic DNA fragment containing the $\sim 1.3-\mathrm{kb}$ promoter region, the entire OSCNGC9 coding region, and the 1458-bp $3^{\prime}$ region was used for vector construction. The fragments were inserted into the binary vector pCAMBIA1305 to generate the pOsCNGC9::OsCNGC9 construct (pGOsCNGC9). Subsequently, the plasmid pGOsCNGC9 was introduced into the Agrobacterium tumefaciens strain EHA105 by electroporation and used to infect the calli of cds1. Rice transformation was performed as previously described. ${ }^{48}$ For CRISPR (Clustered regularly interspaced short palindromic repeats)/Cas9 construction, a 18-bp gene-specific sequence of OsCERK1 or a 18-bp common sequence of OsRLCK185 and OsRLCK55 was synthesized and annealed to form the oligo adaptors. The oligo adaptors were firstly cloned into the entry vector pOs-sgRNA, and then subcloned into the gateway destination vector pOs-Cas9. ${ }^{49}$ The CRISPR/Cas9 plasmids were introduced into Agrobacterium tumefaciens strain EHA105 and then introduced into Kitaake. Positive lines were confirmed by PCR 
followed by sequencing. For the OsCNGC9-OE construct, the fulllength CDS of OsCNGC9 was amplified with PCR method. The fragment was then cloned into the pCUbi1390 vector to generate the OsCNGC9-OE construct, which was then introduced into Kitaake using Agrobacterium-mediated transformation. All transgenic lines were analyzed using the stable $T_{2}-T_{3}$ progenies. All primer sequences for the constructs are listed in Supplementary information, Table S2.

Promoter activity assay

The promoter activity assay was performed as described previously. ${ }^{50} \mathrm{~A} \sim 1.3 \mathrm{~kb}$ promoter region of OsCNGC9 was cloned into the $\mathrm{N}$-terminus of GUS in the expression vector pCAMBIA1305.1 to generate the pOsCNGC9::GUS fusion reporter plasmid, which was introduced into Kitaake by Agrobacteriummediated transformation. Histochemical staining was performed to observe the GUS signal in different tissues and organs in the transgenic plants. Briefly, various tissues of transgenic plants were incubated at $37^{\circ} \mathrm{C}$ in a staining solution containing $100 \mathrm{mM}$ $\mathrm{NaPO}_{4}$ buffer ( $\mathrm{pH}=7.0$ ), $10 \mathrm{mM} \mathrm{Na}$-EDTA, $2 \mathrm{mM}$ X-Gluc, $0.5 \mathrm{mM}$ $\mathrm{K}_{3} \mathrm{Fe}(\mathrm{CN})_{6}, 0.5 \mathrm{mM} \mathrm{K}_{4} \mathrm{Fe}(\mathrm{CN})_{6}$ and $0.1 \%$ Triton X-100. After staining, the samples were incubated with $70 \%$ ethanol until the tissue became clear. All primer sequences for the constructs are listed in Supplementary information, Table S2.

RNA in situ hybridization

Fully expanded leaves were fixed in RNase-free FAA for $12 \mathrm{~h}$ and then dehydrated through an ethanol series. The samples were further embedded in paraffin (Paraplast Plus, Sigma) before sectioning. A cDNA fragment was amplified from the OsCNGC9 coding region and cloned into a pGEM-T vector (Promega) for RNA probe preparation. DIG Northern starter kit (Roche) was used to generate the digoxigenin-labeled RNA probes. In situ hybridization experiments and immunological detection of the signals were performed following the protocol previously described. ${ }^{51}$ Slides were observed under bright field using a Leica DMR microscope (Leica DM5000B), and photographed using a camera fitted with a Micro Color CCD (Apogee Instruments). All primer sequences used here are listed in Supplementary information, Table S2.

Phylogenetic analysis

The full-length amino acid sequence of CNGC family proteins, from rice and Arabidopsis were aligned using DNAMAN software (Lynnon BioSoft, Quebec, Canada). The alignment was adjusted manually and the neighbor-joining tree was constructed by MEGA 4.0 (http://www.megasoftware.net/) using the bootstrap method with 1000 replicates as previously described..$^{52}$ The amino acid sequences were obtained from Rice Genome Automated Annotation System (http://ricegaas.dna.affrc.go.jp/), the Arabidopsis Information Resource (https://www.arabidopsis.org/) and the National Center for Biotechnology Information (https://www. ncbi.nlm.nih.gov/).

Subcellular localization assay

The subcellular localization assay in rice plants was performed as described previously. ${ }^{53}$ The promoter region and the full-length CDS of OsCNGC9 were cloned into the pCAMBIA1305-GFP vector to generate the pOsCNGC9::OsCNGC9-GFP construct for plant transformation. Transgenic roots were used to analyze the subcellular localization of OsCNGC9-GFP fusion protein. The subcellular localization of OsCNGC9 protein in rice protoplasts was performed as described previously. ${ }^{54}$ The full-length CDS of OsCNGC9 was cloned into pA7-GFP vector to form OsCNGC9-GFP fusion protein. A previously characterized plasma membrane protein, PIP2;1 fused with mCherry was used as a plasma membrane marker. ${ }^{55}$ Both constructs were co-transformed into rice protoplasts for subcellular localization analysis. Images were captured using scanning confocal microscope (ZEISS LSM 700). All primer sequences used here are listed in Supplementary information, Table S2.

Protein interaction assay

The $\mathrm{Y} 2 \mathrm{H}$ assay was used to detect protein interaction following the DUALhunter system (Dualsystems Biotech). The full coding sequences of the corresponding genes were fused to the Nub fragment in the pXGY17 vector or to the Cub fragment in the pXGY18 vector, respectively. The process of $\mathrm{Y} 2 \mathrm{H}$ assay was conducted according to the method previously described. ${ }^{30}$

For BiFC assay, the full-length coding sequence of OsCNGC9 was cloned into the binary BiFC vectors PSPYNE173 or PSPYCE(M), respectively. The experiment was conducted as previously described. ${ }^{56}$ Rice GW5 and GSK2 combination was used as a positive control in the BiFC assay. ${ }^{57}$

The luciferase complementation assay was performed in $N$. benthamiana leaves as previously described. ${ }^{58}$ Agrobacteria cells harboring the nLUC and CLUC constructs were co-infiltrated into $N$. benthamiana leaves. Leaves were taken 2 days later, incubated with $1 \mathrm{mM}$ luciferin, and the LUC activity was measured by the NightSHADE LB 985 (Berthold). The interactions with GW5 were used as negative controls in the LUC assay.

For Co-IP assay, protoplasts isolated from WT plants were transfected with desired plasmids, incubated overnight. Total protein was extracted with protein extraction buffer $(150 \mathrm{mM} \mathrm{KCl}$, $50 \mathrm{mM}$ HEPES [pH7.5], 0.4\% Triton-X 100, $1 \mathrm{mM}$ DTT and proteinase inhibitor cocktail). The process of Co-IP assay was conducted as previously described. ${ }^{59}$ Western blots were performed with the anti-HA (CW0092M, CWBIO), anti-GFP (11814460001, Roche), and anti-Mouse secondary antibody (D330, MBL) antibodies. All primer sequences for the constructs are listed in Supplementary information, Table S2.

\section{In vitro phosphorylation assay}

For phosphorylation assay, an in vitro phosphorylation assay method with slight modifications was used. ${ }^{60}$ Briefly, HIS- and MBP-tagged recombinant proteins were expressed in Escherichia coli strain BL21 and purified according to the user's manual, respectively. Phosphorylation assays were performed with purified beads containing OsCNGC9-C-MBP and purified HIS-kinases in 30 $\mu \mathrm{l}$ of kinase buffer (40 mM HEPES [pH 7.5], $20 \mathrm{mM} \mathrm{MgCl} 2,2 \mathrm{mM}$ DTT, $10 \mu \mathrm{Ci}\left[{ }^{32} \mathrm{P}\right]$ YATP, proteinase inhibitor cocktail and phosphatase inhibitor cocktail) for $90 \mathrm{~min}$ at $30^{\circ} \mathrm{C}$, and the reaction was stopped by adding SDS loading buffer. After separation on a $10 \%$ SDS-polyacrylamide gel, the gel was stained with Coomassie blue. Then, the gel was exposed to GE Amersham hyperfilm MP film for detecting phosphorylated proteins.

Phos-Tag assays

Phos-tag assay was carried out as previously described. ${ }^{61}$ Kitaake and Oscerk1 mutant protoplasts expressing OsCNGC9-C-FLAG were treated with chitin for $10 \mathrm{~min}$, and proteins were extracted with protein extraction buffer. The samples were subsequently analyzed using $10 \%$ SDS-polyacrylamide gels with or without $100 \mu \mathrm{M} \mathrm{MnCl} 2$ and $50 \mu \mathrm{M}$ Phos-tag Acrylamide AAL-107 (NARD Institute), respectively. The samples were then transferred to NC membranes, followed by immunoblot analysis with the anti-FLAG (M185-7, MBL) and anti-HSP82 (AbM51099-31-PU, Beijing Protein Innovation) antibodies.

Ion accumulation of $E$. coli assay

Ion accumulation of $E$. coli assay was carried out as previously described with minor modifications. ${ }^{23}$ Briefly, the full-length coding sequence of OsCNGC9 fused with GFP was cloned into PET-30a expression vector (Novagen), and the full-length coding sequence of GFP cloned into PET-30a vector was used as the control. The plasmids were separately transformed into $E$. coli strain DE3 (TransGen Biotech). GFP fluorescence was captured 
after induction with $0.5 \mathrm{mM}$ isopropyl $\beta$-D-thiogalactoside (IPTG) in a $E$. coli bacterial incubation medium containing $2 \times Y T$ medium with $1.6 \%$ Tryptone (OXOID) and $1 \%$ Yeast extract (OXOID). After induction, E. coli cells were collected by centrifugation, pelleted, resuspended in $2 \%$ lactose solution and incubated in $2 \%$ lactose solution containing $1 \mathrm{mM} \mathrm{CaCl}_{2}, 1 \mathrm{mM} \mathrm{RbCl}$ and $1 \mathrm{mM} \mathrm{NaCl}$. After incubation, $E$. coli cells were collected, washed with $2 \%$ lactose solution, and digested in $\mathrm{HNO}_{3}$ overnight. An inductively coupled plasma atomic emission spectroscopy (Varian, Palo Alto, CA) was used for the ion accumulation assay. All primer sequences for the constructs are listed in Supplementary information, Table S2.

Whole-cell patch clamp recordings

OsCNGC9-mediated $\mathrm{Ca}^{2+}$ and $\mathrm{K}^{+}$current recordings in HEK293T cells were performed as described previously. ${ }^{24}$ Briefly, an Axopatch-200B patch clamp setup (Axon Instruments, CA, USA) and a Digidata 1440A digitizer combined to an Olympus IX71 inverted microscope (Japan) were used for whole cell patch clamp experiments. Patch clamping data was acquired and analyzed using the software pClamp10.2 (Axon Instruments, CA, USA). For $\mathrm{Ca}^{2+}$ current recordings in HEK293T cells, the standard bath solution contains $120 \mathrm{mM} \mathrm{NaCl}, 10 \mathrm{mM} \mathrm{CsCl}, 10 \mathrm{mM} \mathrm{CaCl}_{2}, 10 \mathrm{mM}$ HEPES, $2.8 \mathrm{mM} \mathrm{KCl}$ and $2 \mathrm{mM} \mathrm{MgCl} 2$ and the $\mathrm{pH}$ was adjusted to 7.2 with $\mathrm{NaOH}$. The standard pipette solution contains $120 \mathrm{mM}$ Csglutamate, $10 \mathrm{mM}$ HEPES, $8 \mathrm{mM} \mathrm{NaCl}, 6.7 \mathrm{mM}$ EGTA, $3.35 \mathrm{mM}$ $\mathrm{CaCl}_{2}, 3 \mathrm{mM} \mathrm{MgCl}$ and $2.5 \mathrm{mM} \mathrm{Mg-ATP}$ added freshly each day, and the $\mathrm{pH}$ was adjusted to 7.2 with $\mathrm{CsOH}$. The free $\mathrm{Ca}^{2+}$ concentration was $175 \mathrm{nM}$ calculated by the online free software Webmaxc Standard (http://www.stanford.edu/ cpatton/ webmaxc/webmaxcS.htm). The osmolality of bath and pipette solutions was adjusted to $313.3 \mathrm{mmol} / \mathrm{kg}$ with D-glucose. For inward $\mathrm{K}^{+}$current recordings, the bath solution contains $120 \mathrm{mM}$ $\mathrm{NaCl}, 14.5 \mathrm{mM} \mathrm{KCl}, 10 \mathrm{mM}$ glucose, $10 \mathrm{mM} \mathrm{HEPES}, 2 \mathrm{mM} \mathrm{CaCl}_{2}$ and $1 \mathrm{mM} \mathrm{MgCl} 2$ and the $\mathrm{pH}$ was adjusted to 7.2 with $\mathrm{NaOH}$. The pipette solution contains $145 \mathrm{mM}$ K-glutamate, $10 \mathrm{mM}$ HEPES, 8 $\mathrm{mM}$ Na-glutamate, $4 \mathrm{mM}$ Mg-glutamate, $3.35 \mathrm{mM}$ EGTA, $1.675 \mathrm{mM}$ $\mathrm{CaCl}_{2}$ and the $\mathrm{pH}$ was adjusted to 7.2 with $\mathrm{NaOH}$. The free $\mathrm{Ca}^{2+}$ concentration was $100 \mathrm{nM}$ calculated by the online free software Webmaxc Standard. The osmolality of bath and pipette solutions for $\mathrm{K}^{+}$current recordings was adjusted with D-glucose to 313.3 $\mathrm{mmol} / \mathrm{kg}$. primer sequences for the vector construction are listed in Supplementary information, Table S2.

\section{Calcium imaging assay}

Free calcium imaging assay in HEK293T cells was performed as described previously with minor modifications. ${ }^{62}$ Briefly, the fulllength CDS of OsCNGC9 with YC3.6 fused to its C terminus or the full-length CDS of OsRLCK185 (OsRLCK185-K108E) with mCherry fused to its $\mathrm{C}$ terminus was cloned into the $\mathrm{pCl}$-neo vector. The kinase inactive mutant of OsRLCK185, OsRLCK185-K108E was designed according to the method previously described. ${ }^{12}$ YC3.6 fluorescence was observed under an inverted microscope (D1, Carl Zeiss, Germany) for monitoring the ratio $(535 \mathrm{~nm} / 480 \mathrm{~nm})$ of YC3.6. MAG Biosystems 7.5 software (MetaMorph, USA) was used for data acquisition and analysis, and the interval of data acquisition was $9 \mathrm{~s}$. The standard external working solution for $\mathrm{Ca}^{2+}$ imaging contained $120 \mathrm{mM} \mathrm{NaCl}, 10 \mathrm{mM}$ Glucose, $10 \mathrm{mM}$ HEPES, $3 \mathrm{mM} \mathrm{KCl}, 1.2 \mathrm{mM} \mathrm{NaHCO}$ and $1 \mathrm{mM} \mathrm{MgCl}$, pH 7.2 adjusted with $\mathrm{NaOH}$, and external $\mathrm{Ca}^{2+}$ was added as indicated. All primer sequences for vector construction are listed in Supplementary information, Table S2.

Measurements of net $\mathrm{Ca}^{2+}$ flux

Net $\mathrm{Ca}^{2+}$ flux was measured using Non-invasive Micro-test Technique (NMT-YG-100, YoungerUSA LLC, Amherst, MA01002, USA) as previously described with minor modifications. ${ }^{63}$ Leaves sampled from rice seedling were immobilized in the measuring buffer $\left(0.2 \mathrm{mM} \mathrm{CaCl}_{2}, 0.1 \mathrm{mM} \mathrm{NaCl}, 0.1 \mathrm{mM} \mathrm{MgCl}\right.$ and $0.1 \mathrm{mM} \mathrm{KCl}$,
$\mathrm{pH}$ 5.2) for $30 \mathrm{~min}$ equilibration. Then the steady-state fluxes in leaf mesophyll cells were continuously recorded for $5 \mathrm{~min}$ prior to the PAMPs treatments. Chitin (Santa Cruz. Biotechnology) or flg22 (ANASPEC PEPTIDE) peptide was slowly added to the measuring buffer until the chitin concentration reached $10 \mu \mathrm{M}$, or the flg22 concentration reached $10 \mu \mathrm{M}$. Afterward, transient flux of $\mathrm{Ca}^{2+}$ was recorded and continued for $10 \mathrm{~min}$.

Detection of ROS bursts

The detection of ROS bursts was conducted as previously described. ${ }^{64}$ Leaf disks were obtained from fully expanded leaves of rice plants using an ear-hole puncher and floated on $\mathrm{dd}_{2} \mathrm{O}$ for $12 \mathrm{~h}$. Three disks were placed in a $1.5-\mathrm{ml}$ microcentrifuge tube that contains $100 \mu \mathrm{l}$ of luminol (Bio-Rad Immun-Star horseradish peroxidase substrate 170-5040) and $1 \mu \mathrm{l}$ horseradish peroxidase (Jackson Immuno Research) and flg22 or chitin, with $\mathrm{ddH}_{2} \mathrm{O}$ for the control. The tube was immediately measured in a Glomax 20/20 luminometer (Promega) at $15 \mathrm{~s}$ intervals over a period of $30 \mathrm{~min}$.

Detection of PTI-related genes expression

The detection of PTI-related genes expression was conducted as previously described with minor modifications. ${ }^{65}$ Leaves of rice seedlings were cut to $3 \mathrm{~mm}$ strips. The materials were floated on water overnight to recover from wounding stress. Afterward, materials were then treated with water (mock), flg22 peptide or chitin for $1 \mathrm{~h}$ followed by freezing in liquid nitrogen. Materials were used for RNA isolation and qRT-PCR assay. The primer sequences for qRT-PCR assay are listed in Supplementary information, Table S2.

RNA isolation and qRT-PCR assay

The RNA isolation and QRT-PCR assay were conducted as previously described. ${ }^{52}$ Briefly, total RNA was extracted from rice tissues using a plant RNA extraction kit (Qiagen, Hilden, Germany) according to the manufacturer's instructions. For qRT-PCR, total RNA $(1 \mu \mathrm{g})$ was reverse transcribed to cDNA using a QuantiTect Reverse Transcription Kit (Qiagen). The qRT-PCR assay was performed using a SYBR Premix Ex TaqTM kit (TaKaRa) and an ABI prism 7500 Real-Time PCR System. The primer sequences for qRT-PCR assay are listed in Supplementary information, Table S2.

\section{ACKNOWLEDGEMENTS}

We thank Drs. Guo-Liang Wang, Yuese Ning and Xuetao Shi for technical assistance in ROS detection; Drs. Xiaojuan Zhang, Weibing Wang and Jiafan Liu for help in phosphorylation assay. This work was supported by the National Key R\&D Program of China (2017YFD0100404 and 2016YFD0100600) and National Natural Science Foundation of China (31471758).

\section{AUTHOR CONTRIBUTIONS}

Jiachang W., X.L. and A.Z. designed and performed most of the experiments. Y.X., Fan W. and Yihua W. performed genetic analysis and mapping. Yan-Qiu T. and Yong-Fei W. performed the electrophysiological assay. C.L., J.L., S.W., L.M. and H.Z. performed blast inoculation and subcellular localization assay. T.P., Yongfei W. and C.Z. performed protein-protein interaction assay. Xin Z., X.G., L.J., Yupeng W., X.J., Sheng L. and Xiao Z. performed construction and genetic transformation. G. W., X.C. and E. W. constructed the Osrlck185/55 double mutant plants. S.Z., Y.R., Q.L., C.W., Z.C., Jiulin W., Yunlu T. and Shijia L. provided technical help with experiments. Jiachang W., Y.R. and Fuqing W. drafted the manuscript. Jianmin W., J.Z. and H.W. supervised the project and revised the manuscript.

\section{ADDITIONAL INFORMATION}

Supplementary information accompanies for this paper at https://doi.org/10.1038/ s41422-019-0219-7.

Competing interests: The authors declare no competing interests. 


\section{REFERENCES}

1. Jones, J. D. \& Dangl, J. L. The plant immune system. Nature 444, 323-329 (2006).

2. Boller, T. \& He, S. Y. Innate immunity in plants: an arms race between pattern recognition receptors in plants and effectors in microbial pathogens. Science 324, 742-744 (2009)

3. Spoel, S. H. \& Dong, X. N. How do plants achieve immunity? Defence without specialized immune cells. Nat. Rev. Immunol. 12, 89-100 (2012).

4. Couto, D. \& Zipfel, C. Regulation of pattern recognition receptor signalling in plants. Nat. Rev. Immunol. 16, 537-552 (2016).

5. Thor, K. \& Peiter, E. Cytosolic calcium signals elicited by the pathogen-associated molecular pattern flg22 in stomatal guard cells are of an oscillatory nature. New Phytol. 204, 873-881 (2014).

6. Dodd, A. N., Kudla, J. \& Sanders, D. The language of calcium signaling. Annu. Rev. Plant Biol. 61, 593-620 (2010).

7. Boudsocq, M. et al. Differential innate immune signalling via $\mathrm{Ca}^{2+}$ sensor protein kinases. Nature 464, 418-422 (2010).

8. Yuan, P. G., Jauregui, E., Du, L. Q., Tanaka, K. \& Poovaiah, B. W. Calcium signatures and signaling events orchestrate plant-microbe interactions. Curr. Opin. Plant Biol. 38, 173-183 (2017).

9. Liang, X. \& Zhou, J. M. Receptor-like cytoplasmic kinases: central players in plant receptor kinase-mediated signaling. Annu. Rev. Plant Biol. 69, 267-299 (2018).

10. Li, L. et al. The FLS2-associated kinase BIK1 directly phosphorylates the NADPH oxidase RbohD to control plant immunity. Cell Host Microbe 15, 329-338 (2014).

11. Ao, Y. et al. OsCERK1 and OsRLCK176 play important roles in peptidoglycan and chitin signaling in rice innate immunity. Plant J. 80, 1072-1084 (2014).

12. Yamaguchi, K. et al. A receptor-like cytoplasmic kinase targeted by a plant pathogen effector is directly phosphorylated by the chitin receptor and mediates rice immunity. Cell Host Microbe 13, 347-357 (2013).

13. Wang, $C$. et al. OsCERK1-mediated chitin perception and immune signaling requires receptor-like cytoplasmic kinase 185 to activate an MAPK cascade in rice. Mol. Plant 10, 619-633 (2017).

14. Dean, R. A. et al. The genome sequence of the rice blast fungus Magnaporthe grisea. Nature 434, 980-986 (2005).

15. Deng, Y. et al. Epigenetic regulation of antagonistic receptors confers rice blast resistance with yield balance. Science 355, 962-965 (2017).

16. Liu, W., Liu, J., Triplett, L., Leach, J. E. \& Wang, G. L. Novel insights into rice innate immunity against bacterial and fungal pathogens. Annu. Rev. Phytopathol. 52, 213-241 (2014).

17. Chen, X. \& Ronald, P. C. Innate immunity in rice. Trends Plant Sci. 16, 451-459 (2011).

18. Fan, J. et al. The monocot-specific receptor-like kinase SDS2 controls cell death and immunity in rice. Cell Host Microbe 23, 498-510 (2018).

19. You, Q. et al. An E3 ubiquitin ligase-BAG protein module controls plant innate immunity and broad-spectrum disease resistance. Cell Host Microbe 20, 758-769 (2016).

20. Zagotta, W. N. \& Siegelbaum, S. A. Structure and function of cyclic nucleotidegated channels. Annu. Rev. Neurosci. 19, 235-263 (1996).

21. Leng, Q., Mercier, R. W., Hua, B. G., Fromm, H. \& Berkowitz, G. A. Electrophysiological analysis of cloned cyclic nucleotide-gated ion channels. Plant Physiol. 128, 400-410 (2002).

22. Leng, Q., Mercier, R. W., Yao, W. \& Berkowitz, G. A. Cloning and first functional characterization of a plant cyclic nucleotide-gated cation channel. Plant Physiol. 121, 753-761 (1999).

23. Frietsch, S. et al. A cyclic nucleotide-gated channel is essential for polarized tip growth of pollen. Proc. Natl Acad. Sci. USA 104, 14531-14536 (2007).

24. Gao, Q. et al. Arabidopsis CNGC18 is a $\mathrm{Ca}^{2+}$-permeable channel. Mol. Plant 7, 739-743 (2014).

25. Gao, Q. et al. Cyclic nucleotide-gated channel 18 is an essential $\mathrm{Ca}^{2+}$ channel in pollen tube tips for pollen tube guidance to ovules in Arabidopsis. Proc. Natl Acad. Sci. USA 113, 3096-3101 (2016).

26. Pan, Y. et al. Dynamic interactions of plant CNGC subunits and calmodulins drive oscillatory $\mathrm{Ca}^{2+}$ channel activities. Dev. Cell 48, 710-725 (2019).

27. Shih, H. W., DePew, C., Miller, N. \& Monshausen, G. The cyclic nucleotide-gated channel CNGC14 regulates root gravitropism in Arabidopsis thaliana. Curr. Biol. 25, 3119-3125 (2015).

28. Wang, Y. et al. Identification of cyclic GMP-activated nonselective $\mathrm{Ca}^{2+}$-permeable cation channels and associated CNGC5 and CNGC6 genes in Arabidopsis guard cells. Plant Physiol. 163, 578-590 (2013).

29. Wang, Y. et al. CNGC2 is a $\mathrm{Ca}^{2+}$ influx channel that prevents accumulation of apoplastic $\mathrm{Ca}^{2+}$ in the leaf. Plant Physiol. 173, 1342-1354 (2017).

30. $\mathrm{Xu}, \mathrm{Y}$. et al. OsCNGC13 promotes seed-setting rate by facilitating pollen tube growth in stylar tissues. PLoS Genet. 13, e1006906 (2017).
31. DeFalco, T. A., Moeder, W. \& Yoshioka, K. Opening the gates: insights into cyclic nucleotide-gated channel-mediated signaling. Trends Plant Sci. 21, 903-906 (2016).

32. Yu, I. C., Parker, J. \& Bent, A. F. Gene-for-gene disease resistance without the hypersensitive response in Arabidopsis dnd1 mutant. Proc. Natl Acad. Sci. USA 95, 7819-7824 (1998).

33. Yoshioka, K. et al. Environmentally sensitive, SA-dependent defense responses in the cpr22 mutant of Arabidopsis. Plant J. 26, 447-459 (2001).

34. Rodriguez, E., El Ghoul, H., Mundy, J. \& Petersen, M. Making sense of plant autoimmunity and 'negative regulators'. FEBS J. 283, 1385-1391 (2016).

35. Nawaz, Z., Kakar, K. U., Saand, M. A. \& Shu, Q. Y. Cyclic nucleotide-gated ion channel gene family in rice, identification, characterization and experimental analysis of expression response to plant hormones, biotic and abiotic stresses. BMC Genom. 15, 853 (2014).

36. Chiasson, D. M. et al. A quantitative hypermorphic CNGC allele confers ectopic calcium flux and impairs cellular development. eLife 6, e25012 (2017).

37. Schachtman, D. P., Schroeder, J. I., Lucas, W. J., Anderson, J. A. \& Gaber, R. F. Expression of an inward-rectifying potassium channel by the Arabidopsis KAT1 cDNA. Science 258, 1654-1658 (1992).

38. Chinchilla, D. et al. A flagellin-induced complex of the receptor FLS2 and BAK1 initiates plant defence. Nature 448, 497-500 (2007).

39. Wan, J. et al. A LysM receptor-like kinase plays a critical role in chitin signaling and fungal resistance in Arabidopsis. Plant Cell 20, 471-481 (2008).

40. Ranf, S., Eschen-Lippold, L., Pecher, P., Lee, J. \& Scheel, D. Interplay between calcium signalling and early signalling elements during defence responses to microbe- or damage-associated molecular patterns. Plant J. 68 , 100-113 (2011).

41. Ranf, S. et al. Microbe-associated molecular pattern-induced calcium signaling requires the receptor-like cytoplasmic kinases, PBL1 and BIK1. BMC Plant Biol. 14, 374 (2014).

42. Shiu, S. H. et al. Comparative analysis of the receptor-like kinase family in Arabidopsis and rice. Plant Cell 16, 1220-1234 (2004).

43. Zong, $X$. et al. A novel mechanism of modulation of hyperpolarization-activated cyclic nucleotide-gated channels by Src kinase. J. Biol. Chem. 280, 34224-34232 (2005).

44. Jeworutzki, E. et al. Early signaling through the Arabidopsis pattern recognition receptors FLS2 and EFR involves Ca-associated opening of plasma membrane anion channels. Plant J. 62, 367-378 (2010).

45. Dindas, J. et al. AUX1-mediated root hair auxin influx governs $\mathrm{SCF}^{\mathrm{TIR} 1 / \mathrm{AFB}}$-type $\mathrm{Ca}^{2+}$ signaling. Nat. Commun. 9, 1174 (2018).

46. Lolle, S. et al. Matching NLR immune receptors to autoimmunity in camta3 mutants using antimorphic NLR alleles. Cell Host Microbe 21, 518-529 (2017).

47. Li, W. et al. A natural allele of a transcription factor in rice confers broad-spectrum blast resistance. Cell 170, 114-126 (2017).

48. Hiei, Y., Ohta, S., Komari, T. \& Kumashiro, T. Efficient transformation of rice (Oryza sativa L.) mediated by Agrobacterium and sequence analysis of the boundaries of the T-DNA. Plant J. 6, 271-282 (1994).

49. Miao, J. et al. Targeted mutagenesis in rice using CRISPR-Cas system. Cell Res. 23, 1233-1236 (2013)

50. Zhou, F. et al. D14-SCF ${ }^{\mathrm{D} 3}$-dependent degradation of D53 regulates strigolactone signalling. Nature 504, 406-410 (2013).

51. Tan, J. et al. OsCOL10, a CONSTANS-Like gene, functions as a flowering time repressor downstream of Ghd7 in rice. Plant Cell Physiol. 57, 798-812 (2016).

52. Wang, J. et al. Overexpression of OsMYB1R1-VP64 fusion protein increases grain yield in rice by delaying flowering time. FEBS Lett. 590, 3385-3396 (2016).

53. Zhu, S. et al. The OsHAPL1-DTH8-Hd1 complex functions as the transcription regulator to repress heading date in rice. J. Exp. Bot. 68, 553-568 (2017)

54. $\mathrm{Yu}, \mathrm{X}$. et al. A selfish genetic element confers non-Mendelian inheritance in rice. Science 360, 1130-1132 (2018).

55. Lee, H. et al. Drought stress-induced Rma1H1, a RING membrane-anchor E3 ubiquitin ligase homolog, regulates aquaporin levels via ubiquitination in transgenic Arabidopsis plants. Plant Cell 21, 622-641 (2009).

56. Ren, Y. et al. GLUTELIN PRECURSOR ACCUMULATION3 encodes a regulator of postGolgi vesicular traffic essential for vacuolar protein sorting in rice endosperm. Plant Cell 26, 410-425 (2014).

57. Liu, J. et al. GW5 acts in the brassinosteroid signalling pathway to regulate grain width and weight in rice. Nat. Plants 3, 17043 (2017).

58. Liu, J. et al. MicroRNA319-regulated TCPs interact with FBHs and PFT1 to activate CO transcription and control flowering time in Arabidopsis. PLoS Genet. 13, e1006833 (2017).

59. Liang, X. et al. Ligand-triggered de-repression of Arabidopsis heterotrimeric $G$ proteins coupled to immune receptor kinases. Cell Res. 28, 529-543 (2018).

60. Lin, Q. et al. The SnRK2-APC/C ${ }^{\mathrm{TE}}$ regulatory module mediates the antagonistic action of gibberellic acid and abscisic acid pathways. Nat. Commun. 6, 7981 (2015). 
61. Bi, G. et al. Receptor-like cytoplasmic kinases directly link diverse pattern recognition receptors to the activation of mitogen-activated protein kinase cascades in Arabidopsis. Plant Cell 30, 1543-1561 (2018).

62. Yang, D. et al. Calcium pumps and interacting BON1 protein modulate calcium signature, stomatal closure, and plant immunity. Plant Physiol. 175, 424-437 (2017).

63. Ma, Y. et al. COLD1 confers chilling tolerance in rice. Cell 160, 1209-1221 (2015).
64. Shi, X. et al. The fungal pathogen Magnaporthe oryzae suppresses innate immunity by modulating a host potassium channel. PLoS Pathog. 14, e1006878 (2018)

65. Park, C. H. et al. The Magnaporthe oryzae effector AvrPiz-t targets the RING E3 ubiquitin ligase APIP6 to suppress pathogen-associated molecular patterntriggered immunity in rice. Plant Cell 24, 4748-4762 (2012). 